\title{
Comparative metatranscriptomics reveals decline of a neustonic planktonic population
}

\author{
N. Mojib, ${ }^{1, a *}$ M. Thimma, ${ }^{2}$ M. Kumaran, ${ }^{2}$ R. Sougrat, ${ }^{3}$ X. Irigoien ${ }^{1 *}$ \\ ${ }^{1}$ Red Sea Research Center, King Abdullah University of Science and Technology, Thuwal, \\ Saudi Arabia \\ ${ }^{2}$ Biosciences Core Laboratory, King Abdullah University of Science and Technology, Thuwal, \\ Saudi Arabia \\ ${ }^{3}$ Imaging and Characterization Laboratory, King Abdullah University of Science and Technology, \\ Thuwal, Saudi Arabia
}

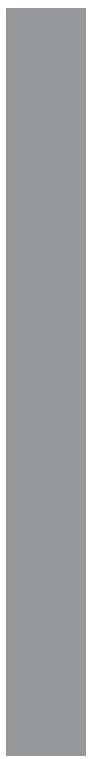

\begin{abstract}
The neuston layer in tropical seas provides a good model to study the effects of increased levels of different stressors (e.g., temperature, ultraviolet radiation and Trichodesmium blooms). Here, we use a comparative in situ metatranscriptomics approach to reveal the functional genomic composition of metabolically active neustonic mesozooplankton community in response to the summer conditions in the Red Sea. The neustonic population exhibited changes in composition and abundance with a significant decline in copepods and appendicularia in July, when Trichodesmium cells were more abundant along with high temperatures and UV-B radiation. Nearly 23,000 genes were differentially expressed at the community level when the metatranscriptomes of the neustonic zooplankton were compared in April, July, and October. On a wider Phylum level, the genes related to oxidative phosphorylation, carbon, nucleotides, amino acids, and lipids were significantly overrepresented in both arthropods and chordates in April and October. On organism level for copepods, expression of genes responsive to oxidative stress, defense against bacteria, immune response, and virus reproduction were increased along with the observed increased appearance of copepod carcasses in the samples collected during July. The differences in expression correspond either to secondary effects of the Trichodesmium bloom or more likely to the increased UV-B radiation in July. Given the dearth of information on the zooplankton gene expression in response to environmental stimuli, our study provides the first transcriptome landscape of the mesozooplankton community during a period of increased mortality of the copepod and appendicularia population.
\end{abstract}

Neustonic plankton are subjected to a higher range of temperatures, higher levels of UV radiation, surface blooms of potentially toxic organisms and higher concentration of contaminants of atmospheric origin than plankton in the

*Correspondence: nazia.mojib@biosci.gatech.edu or Xabier.Irigoyen@ kaust.edu.sa

aPresent address: School of Biological Sciences, Georgia Institute of Technology, Atlanta, Georgia

Additional Supporting Information may be found in the online version of this article.

Data accessibility: RNASeq reads generated using Illumina for the metatranscriptomics data have been submitted to NCBI Short read Archives (SRA) under the Accession nos. SRR1643807, SRR1643809-SRR1643811, and SRR1644043-SRR1644050. deeper layer of the water column (Hardy 1982). Therefore, neuston can be considered a good model to study in situ how increasing levels of multiple stressors could affect at the community and the organisms levels. In particular, neuston layer in tropical areas such as the Red Sea is subjected to high temperatures, high levels of UV radiation (Fig. 1) and occasional blooms of the potentially toxic Trichodesmium sp. (Hawser et al. 1992). These unfavorable conditions limit the use of the upper levels of the water column by the zooplankton affecting the efficiency of food transfer up the food web. Hence, it is critical to understand the possible net effects of multiple stressors on the metabolism of the neustonic mesozooplankton community that play critical roles in the nutrient and biogeochemical cycling in oceans. 


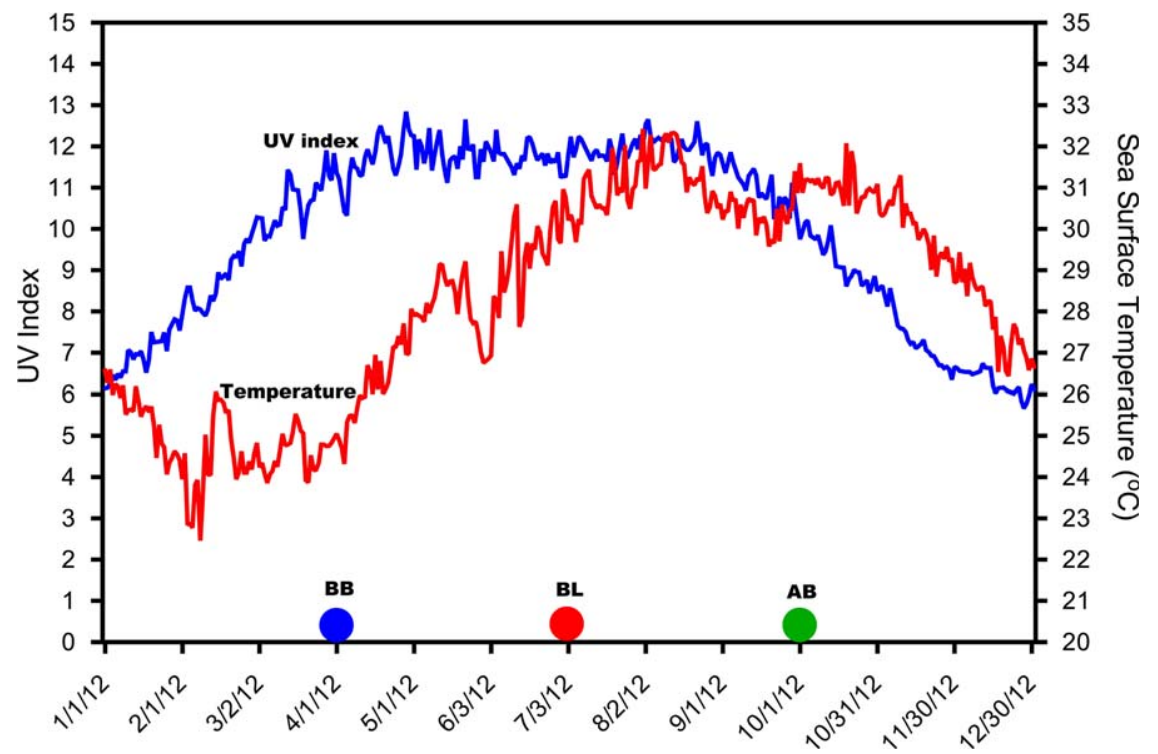

Fig. 1. Variations of the UV index at Mecca station (http://www.temis.nl/uvradiation/index.html) and temperature at $22.4575^{\circ} \mathrm{N}, 39.0305^{\circ} \mathrm{E}$ (http:// coastwatch.pfeg.noaa.gov/erddap/griddap/jplG1SST.html) in 2012. The blue (BB), red (BL), and green (AB) filled circles on $x$-axis indicate the sampling time points. [Color figure can be viewed at wileyonlinelibrary.com]

However, our ability to study in situ the effects of different impacts has been methodologically limited and usually restricted to laboratory incubation experiments assessing one or two stressors at a time. But, the introduction of next-generation sequencing technologies, exponentially increasing sequence databases and ever-evolving data analysis approaches, have changed the situation allowing us to study the combined effect of different stressors at the community and organism level at the same time. It is now possible to understand the molecular functional diversity of natural populations in an ecosystem in a single experiment such as environmental transcriptomics (metatranscriptomics) that provide a less biased view on the eukaryotic gene expression when compared with other approaches (Marchetti et al. 2012; Yadav et al. 2016) . Therefore, we use comparative metatranscriptomics approach to characterize the molecular responses of neustonic mesozooplankton in the Red Sea through summer, a period of potential increased stress. The Red Sea is an oligotrophic environment characterized by very high surface water temperature, salinity, and annual solar radiation (Ngugi et al. 2012). A paired-end Illumina sequencing platform was used to identify the differential abundance of expressed genes from the mesozooplankton collected from the neuston layer in April, July, and October 2012. Our objective was to study the differential responses of organisms from different phyla to the increase in temperature, UV radiation and the presence of Trichodesmium bloom and also to sketch the transcriptomic landscape of the entire mesozooplankton community. We focused on the changes in the gene transcripts in copepods and appendicularia as they were the most affected population. Further, we discussed mortality in copepods based on the transcript and microscopic evidences.

\section{Materials and methods}

Sample collection and metadata

Mesozooplankton were collected from the top $1 \mathrm{~m}$ of the water column using a Manta neuston net (150 $\mu \mathrm{m}$ mesh size) in $10-15$ min long tows in the Red Sea $\left(22.4575^{\circ} \mathrm{N}, 39.0305^{\circ} \mathrm{E}\right)$ in April, July, and October, 2012. For transcriptome analysis, four neuston tows were carried out per sampling date (April, July, and October) to take into account small scale variability. For total RNA extraction, the contents of the cod ends were poured onto a $150 \mu \mathrm{m}$ sieve, washed with filtered seawater and immediately snap-frozen in liquid nitrogen and subsequently stored at $-80^{\circ} \mathrm{C}$. In the laboratory, the zooplankton taxon abundance (per cubic meter) was estimated by counting them under the dissecting microscope (Zeiss Stemi 2000; Carl Zeiss Microscopy) in a $5 \mathrm{~mL}$ subsample (four replicates each) after thoroughly mixing the entire $100 \mathrm{~mL}$ sample of seawater.

Metadata on the Red Sea surface temperature at $22.4575^{\circ} \mathrm{N}$, $39.0305^{\circ} \mathrm{E}$ in 2012 were accessed from National Oceanic and Atmospheric Administration (NOAA) archive database (http:// coastwatch.pfeg.noaa.gov/erddap/griddap/jplG1SST.html). Data of UV indices at Mecca station (close to Red Sea) were taken from the Tropospheric Emissions Monitoring Internet Service (http://www.temis.nl/uvradiation/index.html) that provides global daily analyses of UV indices.

\section{Metatranscriptomics and sequencing}

For total RNA extraction, frozen pellets of the collected mesozooplankton were added directly to Qiazol lysis reagent (Qiagen) and homogenized using RNase-free pestle. Total RNAs were separated and precipitated with chloroform and 
isopropanol, respectively, followed by washing the RNA pellets with $80 \%$ ethanol and redissolved in nuclease-free water. Total RNAs were cleaned and concentrated with RNeasy Mini columns (Qiagen). The isolated RNAs were qualitychecked using a Bioanalyzer (Agilent Technologies) and a NanoDrop (Thermo Scientific) to ensure that the RNA quality index (RQI) values were greater than 8.0 prior to library creation and sequencing by the KAUST Biosciences Core lab. For each sample, one microgram of the total RNA was used to purify mRNA by poly-T oligo-attached magnetic bead selection. Then, mRNAs were converted into cDNAs to prepare RNA-Seq libraries with average insert sizes of 250-300 bp using Truseq ${ }^{\mathrm{TM}}$ RNA Sample Prep Kits (Illumina) following the manufacturer's recommendations. There were four true biological replicates for each sample collected in April (hereafter referred to as collected before Trichodesmium bloom, BB), July (during Trichodesmium bloom, BL), and October (after Trichodesmium bloom, AB) (12 libraries in total) to rule out the impact of expected biological variation. The metatranscriptomes were pair-end sequenced generating 202 bp reads using the HiSeq $^{\mathrm{TM}} 2000$ sequencing system (Illumina) by following standard protocols. A total of 633.23 million raw reads were generated and are available in NCBI Short read Archives (SRA) database (Accession nos. SRR1643807, SRR1643809SRR1643811, and SRR1644043-SRR1644050). Data preprocessing of the generated raw reads and detailed Bioinformatics analyses is provided as Supporting Information (Supplementary Materials and methods; Text S1). Briefly, a total of 339.5 million reads (quality trimmed and rRNA filtered from all 12 libraries) were used to generate the meta assembly using the Trinity software (v2.2.0-released on March 17, 2016) (Haas et al. 2013). A total of 45 taxa of mesozooplankton were identified from the rRNA reads obtained from the metatranscriptome data in this study in the Red Sea (Table S1, Supporting Information) with 357,974 Trinity transcripts and 307,493 Trinity components/genes annotated via Trinotate (v3.0.1) (Tables S2 and S3, Supporting Information). This was followed by abundance estimation, normalization, differential abundance of expressed genes and KEGG pathway analyses (Text S1, Supporting Information). For analysis of results, first we looked at the taxonomic assignment and abundance of transcripts at the wider Phylum level by mapping reads to the meta assembled transcripts. Then as the next step we mapped the community reads to individual transcriptome and genome of two dominant zooplankton species, Acartia fossae and Oikopleura dioica, respectively. This was followed by abundance estimation, normalization, differential expression of genes, and gene enrichment analyses as described in the Supporting Materials and methods (Text S1, Supporting Information).

\section{Transmission electron microscopy}

Whole copepods were fixed with $2.5 \%$ glutaraldehyde. They were then rinsed and post fixed for $1 \mathrm{~h}$ at room temperature in reduced osmium (1:1 mixture of $2 \%$ aqueous osmium tetroxide and 3\% aqueous potassium ferrocyanide) as described previously (Karnovsky 1961). After fixation the copepods were dehydrated in ethanol and processed for Epon embedding. Ultrathin sections (80-100 nm) were cut on an ultramicrotome (Leica UC6), collected on copper grids and stained with lead citrate for $2 \mathrm{~min}$. Sections were then examined with a Titan Krios twin TEM operating at $300 \mathrm{kV}$.

\section{Results}

\section{Community composition}

In April when the Red Sea surface temperature was $26^{\circ} \mathrm{C}$ and UV index 11 (Fig. 1), copepods appeared to be the most abundant group followed by appendicularia based on microscopic examinations. Their average abundance in April was 3307 and 1463 individuals $\mathrm{m}^{-3}$, representing 56\% and 25\% of the total zooplankton community, respectively (Fig. 2a). In July, when the Red Sea surface temperature reached $31^{\circ} \mathrm{C}$ and UV index upto 12 maximum, the neustonic plankton assemblage shifted to one dominated by the Trichodesmium erythraeum colonies (confirmed by $16 \mathrm{~S}$ rRNA sequences obtained in the metatranscriptome generated in this study among the rRNA sequences sorted by SortMe RNA software) exhibiting a raft morphology with an abundance of $\sim 1.44 \times$ $10^{4}\left(\mathrm{SD} \pm 0.2 \times 10^{4}\right)$ trichomes per liter. Trichodesmium was found in the samples from June to September, but in July, 2012, the Trichodesmium bloom was intense; cells looked healthy and showed no signs of degradation. Under these conditions in July, the copepod and appendicularia population densities decreased to 490 and 231 individuals $\mathrm{m}^{-3}$, respectively, with the appearance of copepod carcasses ( $\sim 60 \%$ of the total copepods) in the sample. It is to be noted that in July, Macrosetella sp. (harpacticoid copepods known to graze on Trichodesmium sp.) (Roman 1978; Hawser et al. 1992) appeared in noticeable numbers. In October (temperature still around $30^{\circ} \mathrm{C}$ and UV index reduced to 8-9; Fig. 1 and no Trichodesmium bloom) the community composition reverted to the original numbers. Other mesozooplankton organisms such as molluscan population density increased gradually in July and October compared with April. Other meroplanktonic larvae appeared to maintain a constant population density throughout the sampling period.

The mesozooplankton community response was investigated using a comparative in situ metatranscriptomics approach that allowed studying changes in both species composition and gene expression patterns. We found that approximately $65.3 \%$ of our mRNA sequence reads (all 12 mRNA libraries) mapped to the meta assembled contigs/transcripts. The ratio of used mRNA sequence reads for quantitative gene expression profiling in our study was comparable and better than most of the recent metatranscriptomics analyses approaches (0.5-65\%) (Urich et al. 2008; Stewart et al. 2011; Marchetti et al. 2012; Radax et al. 2012; Ishii et al. 2013). Owing to relatively low number of annotated 
a Relative population density
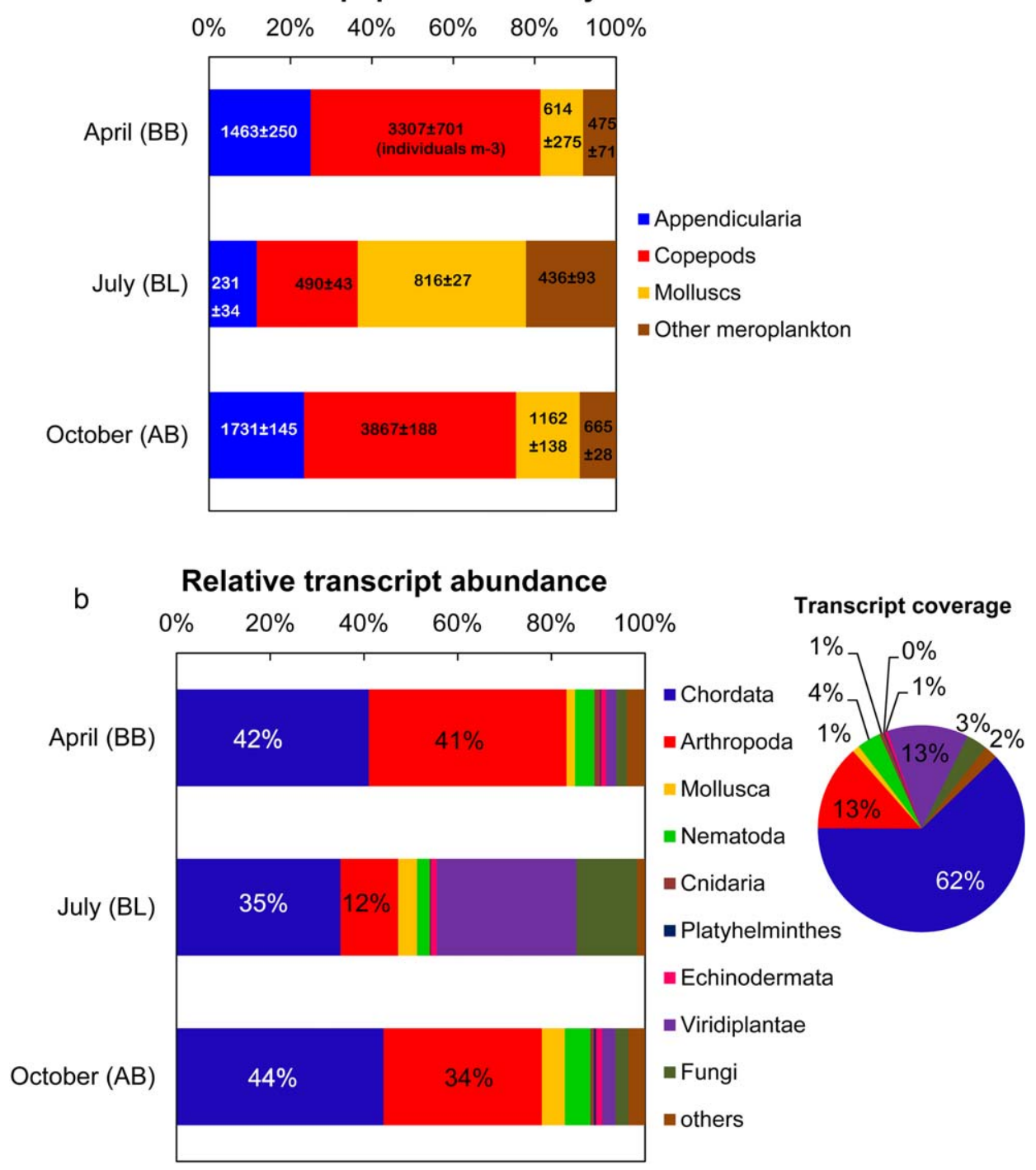

Fig. 2. Community composition of the neustonic zooplankton. (a) Relative density of the total zooplankton as enumerated under the microscope in April (BB, before Trichodesmium bloom), July (BL, during bloom), and October (AB, after bloom) in the Red Sea. The exact population density is indicated on each bar as individuals $\mathrm{m}^{-3} \pm$ standard deviation. (b) Comparative Phylum-based relative eukaryotic transcript abundance of expressed genes in April (BB), July (BL), and October (AB) in the Red Sea. The total number of transcripts annotated to different Phyla (transcript coverage) is depicted as pie chart with relative percentages. [Color figure can be viewed at wileyonlinelibrary.com]

sequences from zooplankton taxonomic categories in the current databases, each meta assembled transcripts were not assigned to each individual present in the meta community. Therefore, we performed analysis of abundance of taxaspecific eukaryotic transcripts on a broader Phylum level to look at the community composition at the transcript level. This resulted in results comparable to the microscopic analysis. Phylum Chordata and Arthropoda comprised 62\% and $13 \%$ of the total annotated eukaryotic transcripts as shown in the transcript coverage pie chart in Figure $2 \mathrm{~b}$. When we looked at the relative abundance of each expressed gene (overall expression of all transcripts of a given gene) in respective Phyla, it was observed that in April Phylum Chordata and Arthropoda dominated the total expressed genes, averaging $41 \%$ and $42 \%$, respectively (Fig. 2 b). In July, abundance of expressed genes in chordates decreased to 35\% and that of arthropods declined to $12 \%$. Abundance of expressed genes from Phyla Viridiplantae and Fungi increased to 30 and 13\%, respectively. Increased abundance of Viridiplantae and Fungi could be explained by the fact that Trichodesmium colonies form raft like structure and acts like a substratum for the growth of organisms from these groups (fungi and Viridiplantae) (Hewson et al. 2009) and $150 \mu \mathrm{m}$ meshes could not filter these rather large-sized Trichodesmium rafts. 


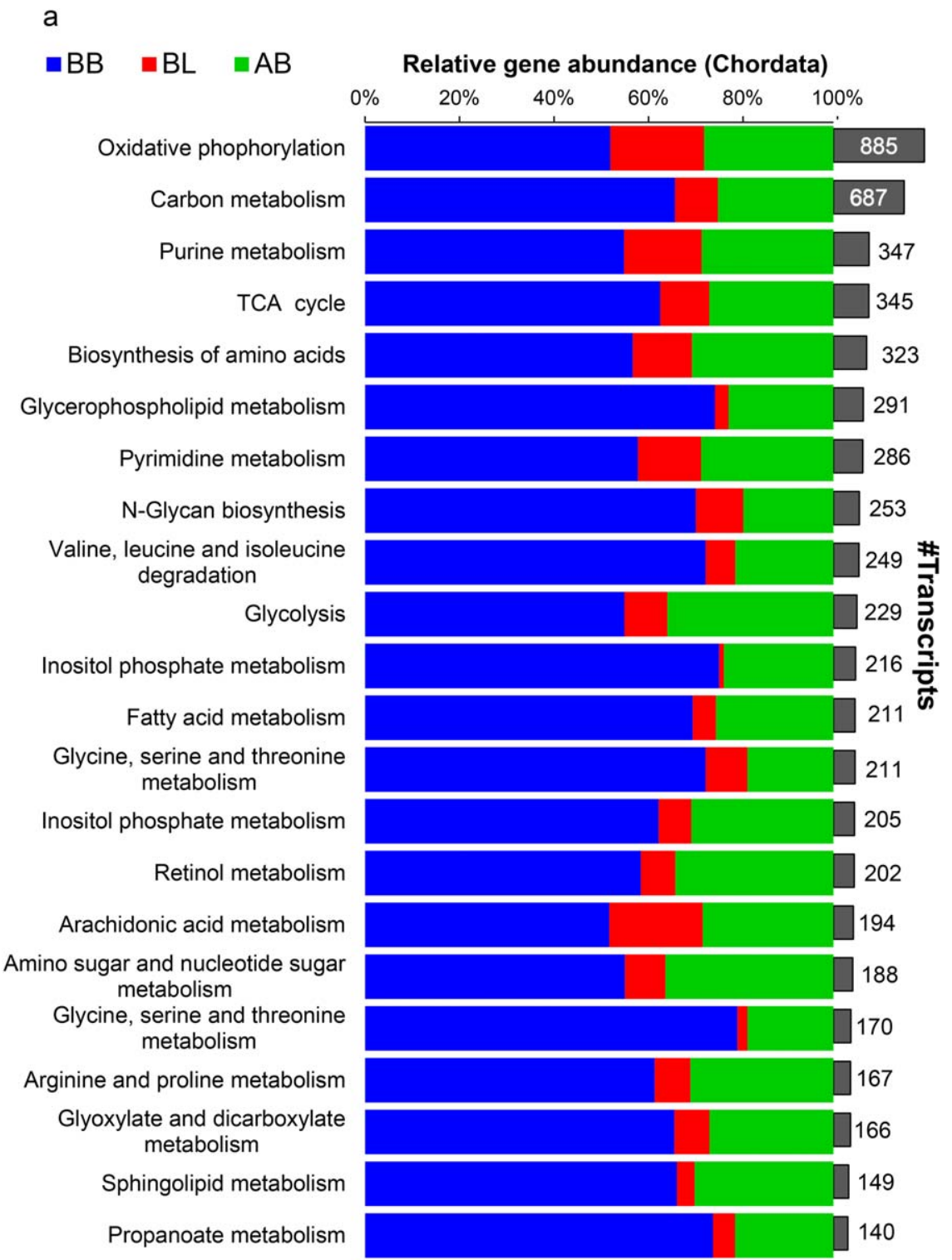

Fig. 3. Taxa-specific relative gene abundance based on KEGG orthology. The stacked bars represent relative gene abundance for Chordata (a) and (b), (next page), Arthropoda in April (BB), July (BL), and October (AB). Each bar represents the distribution of the collection of transcripts assigned to predicted genes in a specific metabolic pathway on the basis of homology (BLASTx, e-value $\leq 10^{-3}$ ) and annotation via KEGG orthology in the BB (blue), $B L$ (red), and $A B$ (green) libraries. The metabolic pathways are arranged in decreasing order of the number of transcripts identified with oxidative phosphorylation containing the highest number of transcripts as depicted in the bar graph on the right panel. [Color figure can be viewed at wileyonlinelibrary.com]

Consequently, we observed transcripts from these phyla in the libraries from July when Trichodesmium colonies were present. There was slight increase in the abundance of gene expression in Phylum Mollusca in July and October indicating that their population is not affected in July as compared with arthropods. In October, the relative community composition reverted nearly to the same as observed during April with chordates being $41 \%$ and arthropods increasing to $34 \%$ suggesting that their population was on the way to recovery. Further, integration of the observed taxa (population density as observed under the microscope) with taxa-specific genes in all 3 months suggested that among chordates, appendicularia from the subphylum Tunicata and among arthropods, copepods were the most affected population in July.

\section{Metabolic landscape of the zooplankton community}

To investigate further, we decided to look into the metabolic landscape (by KEGG orthology) of the two Phyla (Chordata and Arthropoda) based on the abundance of expressed genes (Fig. 3a,b). In April (BB) and October (AB), 


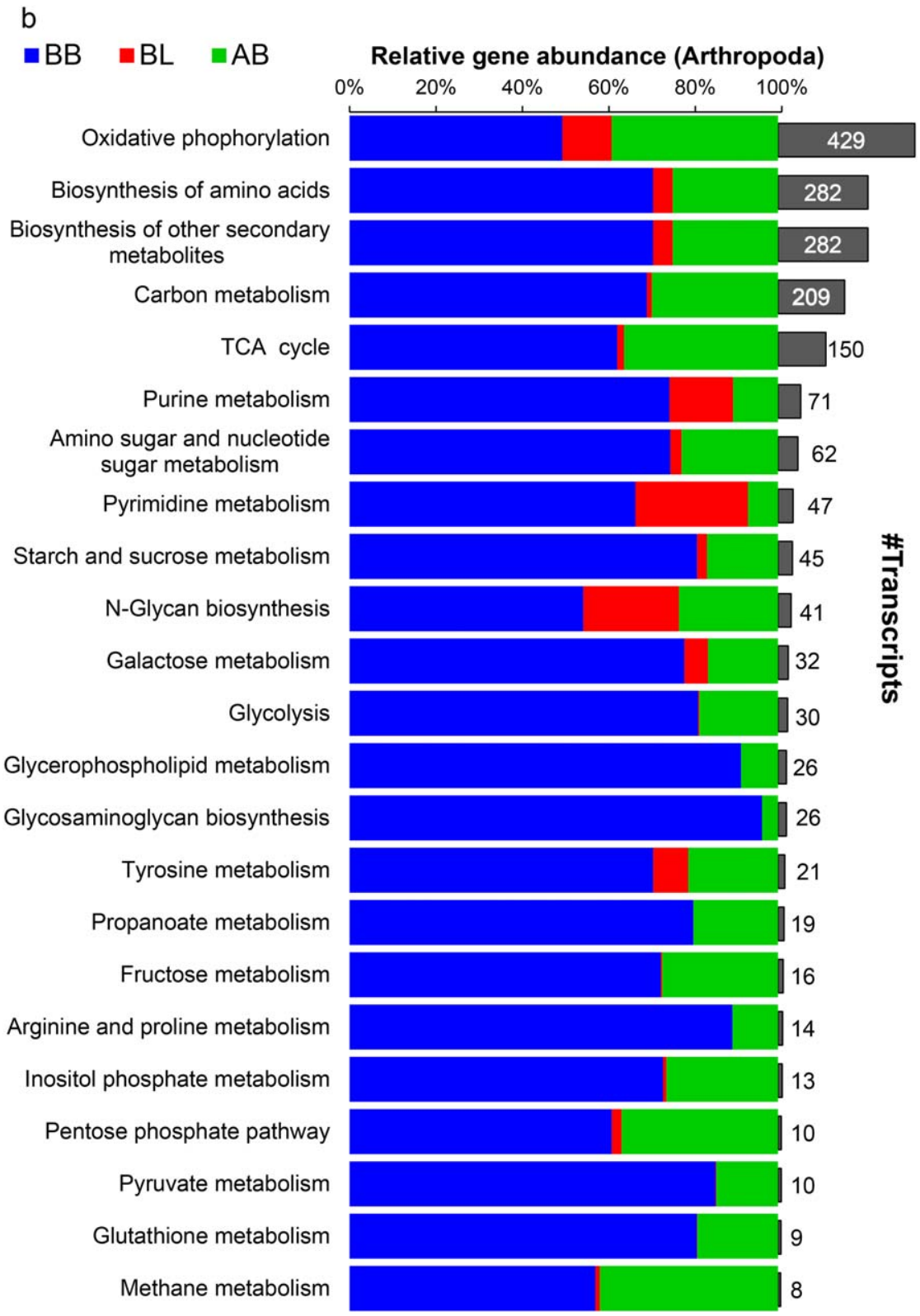

Fig. 3. (Continued).

both chordates and arthropods were largely engaged in oxidative phosphorylation, carbon metabolism (TCA cycle, glycolysis/gluconeogenesis), nucleotide metabolism (purine and pyrimidine), amino acid metabolism, and lipid metabolism. For chordates, all of their major metabolic activities were decreased in July (BL) when compared with April and then increased in October (AB) (Fig. 3a). For arthropods most of their essential metabolic activities (oxidative phosphorylation, TCA cycle, glycolysis/gluconeogenesis, and nucleotide metabolism) were either absent or decreased drastically in July (BL) (Fig. 3b) indicating that at least the copepods (dominating the Arthropoda assigned transcripts) remaining at the surface were dying. This result corroborated the previous observation of copepod carcasses comprising almost $60 \%$ of the total copepods in the samples that were collected from the neuston in July. Further examination of the set of genes expressed in oxidative phosphorylation under three conditions in chordates and arthropods (Fig. 4) it was found that there was decrease in the gene expression of all the series of protein complexes that transfer electrons from $\mathrm{NADH}$ (or $\mathrm{FADH}_{2}$ ) to $\mathrm{O}_{2}$ via redox reactions in July. This electron transport chain includes NADH dehydrogenase (complex I), fumarate dehydrogenase (complex II), cytochrome reductase (complex III), and cytochrome oxidase (complex IV). In this 


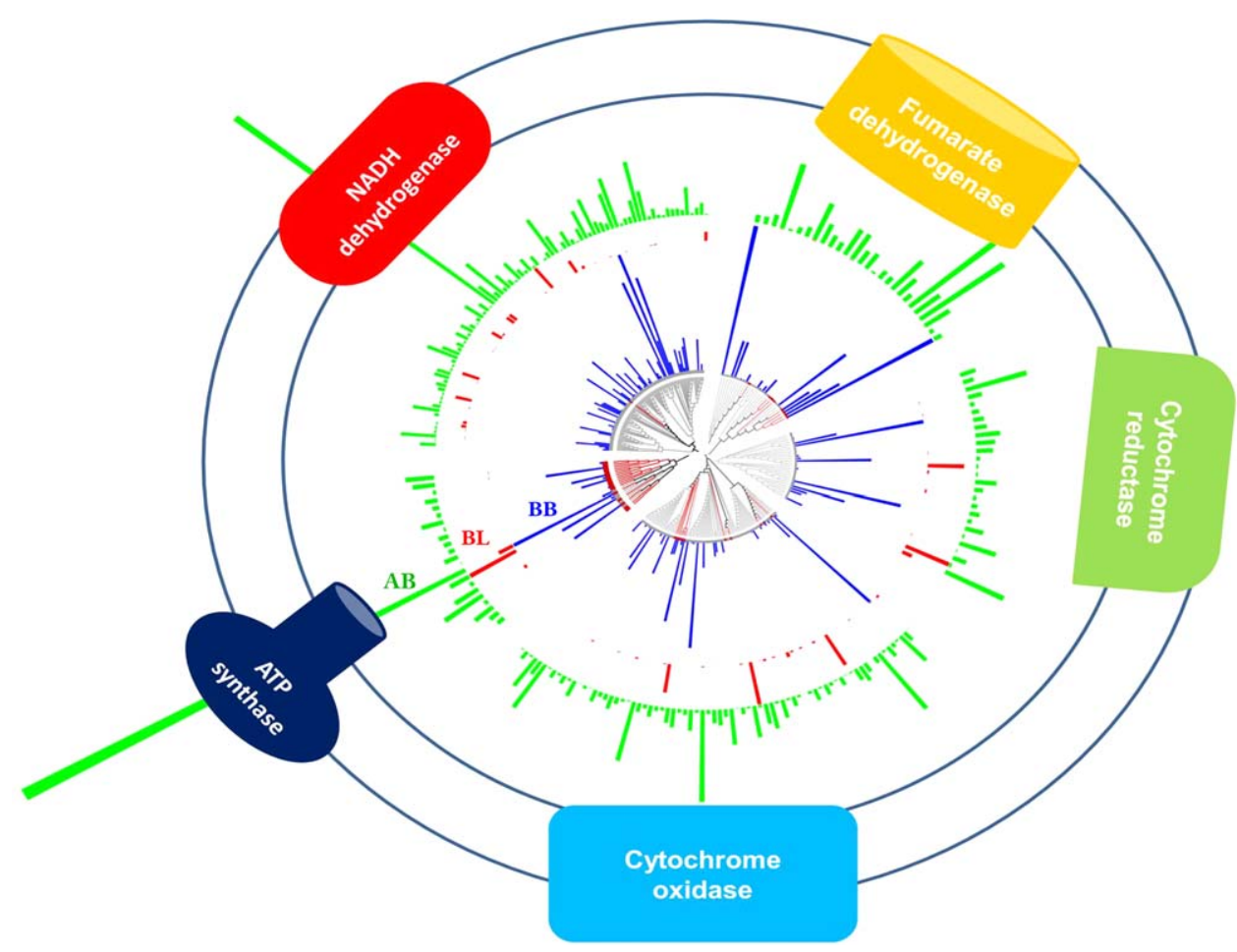

Fig. 4. Comparison of differential abundance of expressed genes related to mitochondrial oxidative phosphorylation in samples from April (BB), July $(B L)$, and October $(A B)$. The radial bar graphs show fragments per kilobase of transcript per million mapped reads (FPKM) values of each differentially expressed transcripts. The red branches in the phylogenetic tree indicate transcripts that belong to arthropods and gray branches indicate transcripts that belong to chordates. [Color figure can be viewed at wileyonlinelibrary.com]

study, it was found that in July (BL) there was relatively more gene expression of NADH-dehydrogenase (complex I) when compared with other protein complexes which may suggest that electrons could be delivered to the respiratory chain. However, absence of gene expression of complex II indicate that this protein complex was inhibited and thus that the respiratory chain was blocked and electrons were undeliverable. Overall, in July oxidative phosphorylation or respiration was affected in both Phyla.

\section{Differential abundance of expressed genes of the zooplankton community}

In addition to transcript abundance calculation by mapping the metatranscriptome reads to the meta assembled transcripts, the trimmed mean of $M$ values (TMM) normalization method was applied to minimize the impacts of changes in species abundances on inferring the abundance of differentially expressed genes across all the samples (Marchetti et al. 2012) (Fig. S1, Supporting Information). Pairwise comparison of the zooplankton community differential abundance of expressed genes between the 2 months (BB vs. $\mathrm{AB}$; $\mathrm{BB}$ vs. $\mathrm{BL}$; and $\mathrm{AB}$ vs. $\mathrm{BL}$ ) revealed significant differences in their transcript profiles (Figs. S2 and S3, Supporting Information). The hierarchical clustering ( $p$ value $<10^{-4}$ ), where the transcripts with similar abundance patterns across the samples at the community level were grouped together, also exhibited around 23,251 significant differentially abundant expressed transcripts in all 3 months (Fig. S4, Supporting Information). Then, we performed a detailed analysis upon splitting the data set as shown in Supporting Information Fig. S4 into sets of transcripts with related abundance patterns by partitioning the hierarchically clustered transcript tree (Fig. S5, Supporting Information). This generated the subclusters containing an abundance matrix showing the gene expression in 3 months (April, July, and October). We further analyzed transcripts from subcluster 2 that were more abundantly expressed in July (BL) relative to April and October by assigning gene ontology (GO) function to each gene (Table S6, Supporting Information). Proteolysis and nucleosome assembly were highly abundant in chordates in July, in addition to genes responsive to stimuli or stress, virus reproduction and immune response. In arthropods (mainly composed of copepods), genes responsive to oxidative stress, defense against bacteria, immune response and virus reproduction were also abundant along with the genes involved in translation and respiration. In molluscs, genes responsive to translation, respiration, proteolysis, iron transport, neurotoxin as well as host-virus interactions were increased in July (Table S6, Supporting Information). Further, the genes involved in pathways relating to oxidative phosphorylation (electron transport in the mitochondria) show an increased abundance in April and October samples 
a

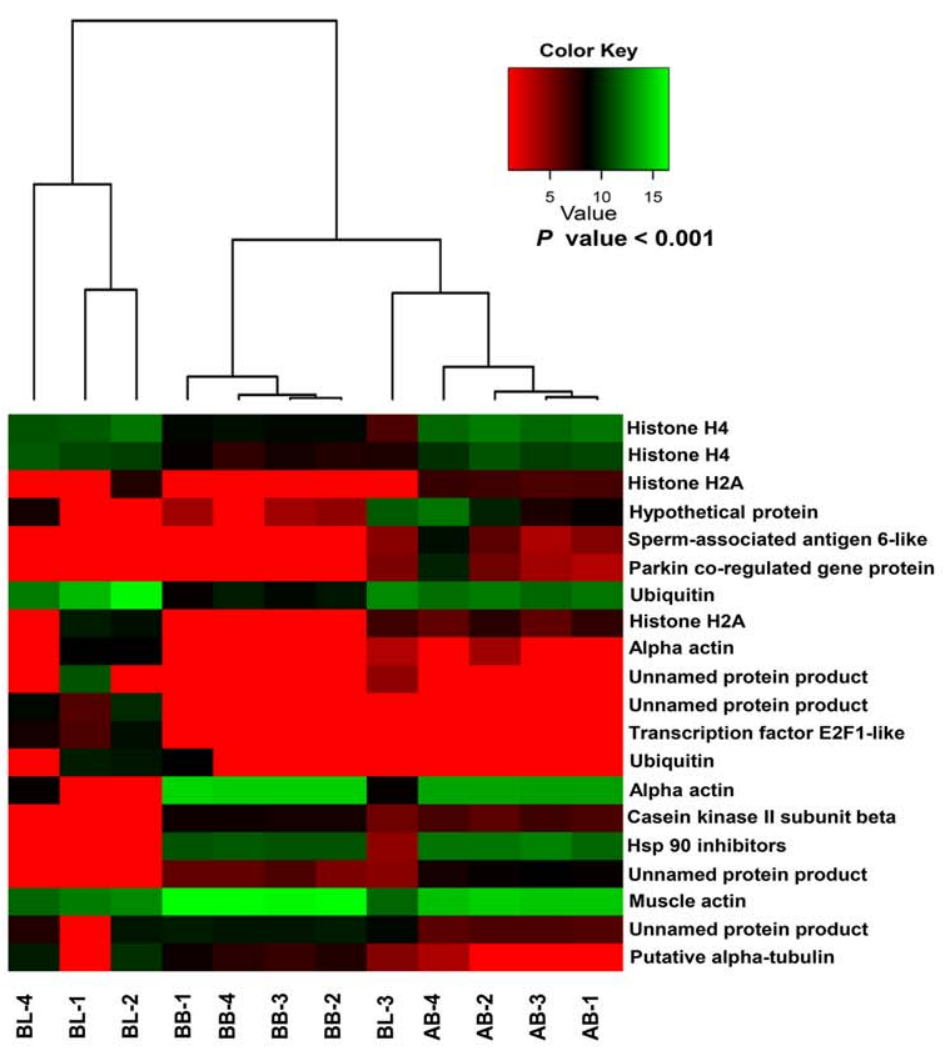

b

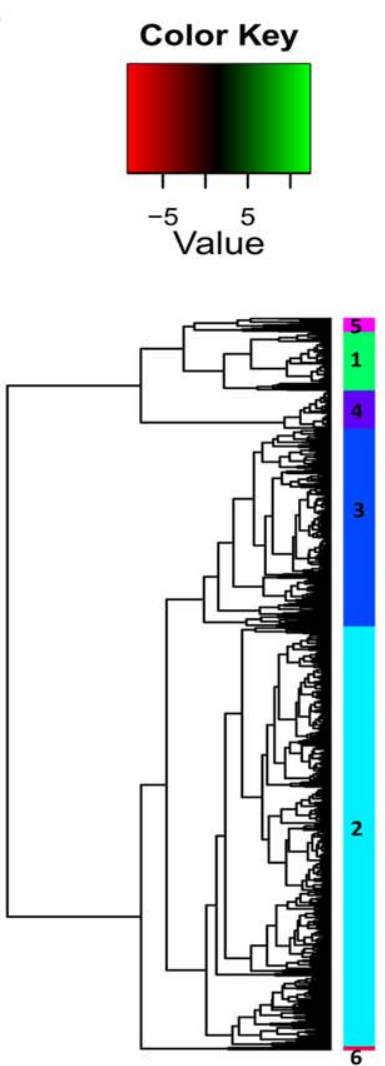

$P$ value $<0.001$
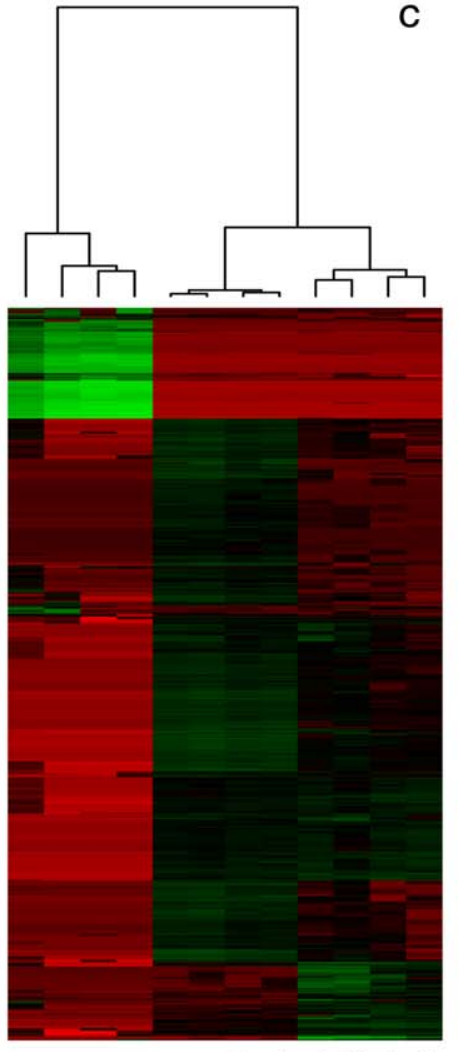

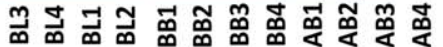

C GO enriched terms in cluster 1,4 \& 5 (BL replicates)

\begin{tabular}{|c|c|c|}
\hline GO-ID & Term & $P$-Value \\
\hline GO:0016032 & viral reproduction & $2.35 \mathrm{E}-21$ \\
\hline GO:0051704 & multi-organism process & $1.21 \mathrm{E}-15$ \\
\hline GO:0044419 & interspecies interaction & $1.21 \mathrm{E}-15$ \\
\hline GO:0044403 & symbiosis & $1.21 \mathrm{E}-15$ \\
\hline GO:0000003 & reproduction & $7.69 \mathrm{E}-12$ \\
\hline GO:0008135 & $\begin{array}{l}\text { translation factor activity, } \\
\text { nucleic acid binding }\end{array}$ & 0.0118027 \\
\hline GO:0008233 & peptidase activity & 0.0183014 \\
\hline \multicolumn{3}{|c|}{ GO enriched terms in cluster 2 (BB \& AB replicates) } \\
\hline GO-ID & Term & $P$-Value \\
\hline GO:0006091 & precursor metabolites and energy & $9.01 \mathrm{E}-18$ \\
\hline GO:0007010 & cytoskeleton organization & $1.04 \mathrm{E}-06$ \\
\hline GO:0009056 & catabolic process & $1.21 \mathrm{E}-05$ \\
\hline GO:0009653 & anatomical structure morphogenesis & $1.56 \mathrm{E}-04$ \\
\hline GO:0000003 & reproduction & 4.23E-04 \\
\hline GO:0030154 & cell differentiation & $7.65 \mathrm{E}-04$ \\
\hline GO:0006412 & translation & 0.0018771 \\
\hline GO:0007275 & multicellular organismal development & 0.0027905 \\
\hline GO:0007028 & cytoplasm organization & 0.0046726 \\
\hline GO:0003774 & motor activity & $1.00 \mathrm{E}-07$ \\
\hline GO:0005198 & structural molecule activity & 1.97E-06 \\
\hline GO:0000166 & nucleotide binding & $2.94 \mathrm{E}-06$ \\
\hline GO:0045735 & nutrient reservoir activity & $3.48 \mathrm{E}-04$ \\
\hline GO:0009055 & electron carrier activity & 0.0018771 \\
\hline GO:0005215 & transporter activity & 0.0075893 \\
\hline \multicolumn{3}{|c|}{ GO enriched terms in cluster 3 (BB replicates) } \\
\hline GO-ID & Term & $P$-Value \\
\hline GO:0007049 & cell cycle & $2.86 \mathrm{E}-04$ \\
\hline GO:0006259 & DNA metabolic process & 0.0010336 \\
\hline GO:0008219 & cell death & 0.0017111 \\
\hline GO:0006950 & response to stress & 0.003226 \\
\hline
\end{tabular}

Fig. 5. 
Fig. 5. (See previous page) Differential gene expression analysis of (a) $O$. dioica (appendicularia) across all samples from April (BB), July (BL), and October (AB) $\left(p\right.$ value $\left.<10^{-3}\right)$ is shown by heatmap of 20 differentially expressed genes. (b) of $A$. fossae (copepod) across all samples $\left(p\right.$ value $\left.<10^{-3}\right)$, depicted by a heatmap showing 888 differentially expressed genes in the copepod A. fossae across all the samples, April (BB), July (BL), and October (AB). Samples and genes (columns and rows, respectively) are reordered on the basis of the average value of gene expression (log 2 ratio) leading to clustering of groups of genes and samples with similar average expression levels according to the color key on the top. (c) The GO enrichment analyses indicating the GO term for the enriched biological process in different clusters of differentially expressed genes along with the $p$ values in $A$. fossae. [Color figure can be viewed at wileyonlinelibrary.com]

for both chordates and arthropods (Fig. 4). This increased oxidative phosphorylation within the electron transport chain, which is responsible for the majority of ATP (Adenosine triphosphate) production (a marker for active respiration) and ROS (reactive oxygen species) formation (marker of stress) could be a good indicator of their metabolic activities in an ecosystem under given conditions. Moreover, zooplankton community respiration in the ocean is a key variable in resolving heterotrophic state of the ocean ecosystems.

\section{Response of appendicularians and molluscs-single genome mapping approach}

The gene activities of the appendicularians were tracked by mapping all 12 metatranscriptome libraries generated from the neustonic zooplankton communities to the $O$. dioica genome (Accession no. ASM20953v1) (Denoeud et al. 2010). Out of 17,512 genes, $31.3 \%$ and $27.7 \%$ genes were found to be expressed in April and October, respectively, whereas only $4.4 \%$ of the genes were expressed in July (Fig. S6, Supporting Information). The hierarchical clustering ( $p$ value $<10^{-3}$ ), as depicted by a heatmap, exhibited 20 differentially expressed Oikopleura genes (Fig. 5a). Out of these, expression of ubiquitin protein was increased several folds during the bloom in July and sustained its expression after the bloom demise in October. The increased levels of ubiquitin conjugates generally indicate mild-oxidative stress, indicating that appendicularians were in mild oxidative stress during July and continued to be in stress in October. The expression of $\mathrm{H} 2 \mathrm{~A}$ and $\mathrm{H} 4$ were also increased in July and continued to be expressed in October, and perhaps are related to DNA replication and cell division. The significance of increased expression of gene such as HSP90 inhibitor and reproduction-related sperm-associated antigen 6 like cannot be explained. On the other hand, similar mapping of reads to the Crassostrea gigas genome representing Phylum Mollusca (Accession no. AFTI01000000) (Zhang et al. 2012) revealed that mollusc population dynamics was not affected in July as observed earlier (Fig. S7, Supporting Information). Only one gene, selenide water dikinase, was found to be differentially expressed during July (when April and July transcriptomes were compared). This gene is reported to play roles in metabolism and oxidative stress defense.

\section{Response of copepods- $A$. fossae transcriptome mapping approach}

The observation of carcasses as well as reduced metabolic activities of copepods led us to further investigate the observed copepod mortality in July. We mapped 12 metatranscriptome libraries generated from the neustonic zooplankton through the study period to our in-house reference transcriptome (Mojib et al. 2014) generated from one of the dominant copepod species, A. fossae, found in the neuston layer of the a

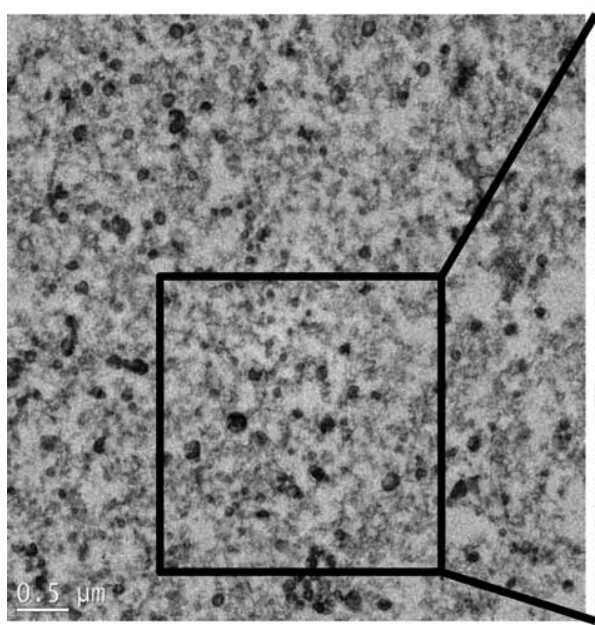

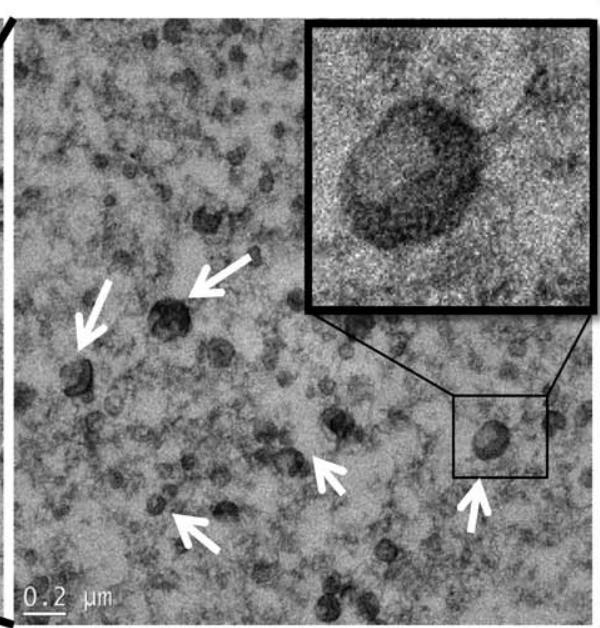

b

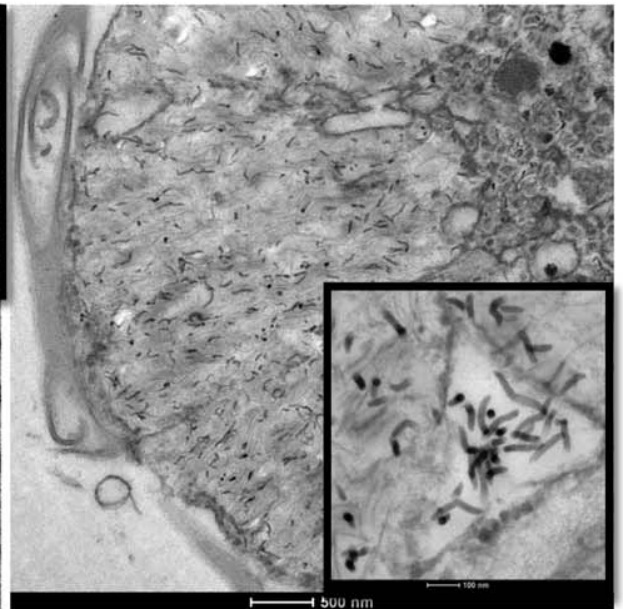

Fig. 6. Transmission electron micrographs at various magnifications (a) showing virus-like particles (scale, $0.5 \mu \mathrm{m}$ ) with a zoom in the micrograph (scale, $0.2 \mu \mathrm{m}$ ) (indicated by bold white arrows) in the connective tissue of $A$. fossae collected during the Trichodesmium bloom in July; (b) virus-like particles in Trichodesmium cells during the bloom in July (scale, $0.5 \mu \mathrm{m}$ ); inset (scale, $0.1 \mu \mathrm{m}$ ). 
Red Sea. The hierarchical clustering $\left(p\right.$ value $\left.<10^{-3}\right)$ as depicted by heatmap exhibited around 888 significant $A$. fossae differentially expressed genes across all 3 months (Fig. 5b). The GO enrichment analyses in April and October (cluster 2 and 3; Fig. 5c) exhibited genes enriched in translation, cell cycle, energy metabolism, cytoskeletal organization, multicellular organismal development, and electron carrier activity as observed earlier. The GO enrichment analyses on the cluster 1,4 , and 5 where genes were highly expressed in July exhibited enrichment of genes involved in viral reproduction, interspecific interactions, multiorganism process, and symbiosis. Although this study was not designed originally to look into viruses as a factor involved in the copepod mortality, the enrichment of genes involved in viral reproduction raised the question of viral presence in the copepods. Therefore we carried out a preliminary investigation of the presence of viruses in the tissues of copepods using transmission electron microscopy (TEM). The micrographs of the connective tissues of the copepod $A$. fossae isolated from the sample collected during July indicated the presence of virus-like particles (VLPs) (Fig. 6a, Fig. S8a-h, Supporting Information). The VLPs observed in the copepod tissues range in sizes from 50 to $200 \mathrm{~nm}$ (Fig. $6 a)$. In contrast, we did not find VLPs in the TEM images of the connective tissues of $A$. fossae collected in April. To rule out the suggestion that VLPs observed in copepods did not originate from the ingested Trichodesmium cells, we also analyzed the morphology of VLPs in the Trichodesmium cells collected from the bloom samples. The TEM images showed that the VLPs in Trichodesmium cells had different morphologies from those observed in the tissues of the copepod $A$. fossae (Fig. 6b, Fig. S8i-k, Supporting Information).

\section{Discussion}

Our results show a distinct decline in the abundance of copepods and appendicularia in the neuston during the month of July in comparison to April and October. When we examined the transcripts assigned to broad taxonomic categories (Phylum-level) by mapping to meta assembled zooplankton transcripts across 3 months with conditions of before, during and after the Trichodesmium bloom, nearly twenty three thousand genes were differentially expressed at the community level. In both chordates and arthropods, genes related to the metabolisms of energy, carbon, nucleotides, amino acids and lipids were overrepresented in April and October. In particular, zooplankton community respiration as indicated by the increased oxidative phosphorylation in April and October (Fig. 4) suggests enhanced heterotrophic energy production. Whereas, in July during the Trichodesmium bloom, genes responsive to stimuli, oxidative stress or viruses and genes related to immune response were increased indicating that both chordates and arthropods were under stress. Further presence of copepod carcasses and the relative decline in the abundance of transcripts of Phylum Arthropoda (analyzed from meta assembly data) and specifically one representative copepod species $A$. fossae (analyzed by mapping to its transcriptome), is suggestive of enhanced mortality of copepods in the neuston layer. The neuston appendicularian population density also decreased during July. This decline of the copepod and appendicularian population in the neuston can be explained by two non-exclusive hypotheses: first, a fraction of the population perished; second, a fraction of them migrated into deeper waters to escape an adverse environment. In either case, both mechanisms result in a significant decrease in potential grazers in the neuston layer where Trichodesmium grew.

In the light of above results, three potential hypotheses about the causes of mortality in copepods can be postulated: (1) direct toxic effect of Trichodesmium, (2) an indirect effect through a weakening of the immune system that might facilitate viral infection, and (3) other effects, not related to Trichodesmium, such as temperature or UV radiation. Extracts of some species of Trichodesmium have shown to be toxic for calanoid copepods (Hawser et al. 1992; Guo and Tester 1994), but the ingestion of healthy cells when no other food was available did not appear to be toxic (Guo and Tester 1994). Actually it is unlikely that calanoid copepods ingest Trichodesmium under normal conditions ( $\mathrm{O}^{\prime} \mathrm{Neil}$ and Roman 1994). Further, our differential expression data (obtained by A. fossae transcriptome mapping approach) do not show an increase in the expression of genes potentially related to toxicity. Therefore a direct toxic effect of Trichodesmium through ingestion seems to be an unlikely reason to explain the decline of the copepods in the neuston. An alternative possibility is that although not ingested, the presence of Trichodesmium derived compounds in the water may weaken the organism as observed by Guo and Tester (1994) and facilitate the spread of viral infection and consequent mortality. To our knowledge only one previous study demonstrated the presence of VLPs in the connective tissues of natural population of calanoid copepods (Dunlap et al. 2013). Here, the microscopy-based in situ occurrence of virus-like particles in the copepod tissues and the many transcripts annotated to viruses in our metatranscriptomics data from the community samples collected in July (data not shown) strongly suggest an important role of viruses in the observed mortality. The metatranscriptome data alone cannot confirm that the copepods are infected with viruses but the microscopic observations provide us a lead to further examine the potential link between copepod mortality and viral infection as it adds a new dimension to the complex trophic interactions established among prey, predators, and pathogens.

Two other factors that change significantly during our study and could have an effect on zooplankton community are temperature and UV radiation (Fig. 1). From April to July Red sea surface temperature increases from 26 to $30-31^{\circ} \mathrm{C}$ (Raitsos et al. 2011). However, in October, when the neustonic community was already recovered, temperatures were 
still around $30^{\circ} \mathrm{C}$. In the differential gene expression analysis we did not find an increase in the expression of heat shock proteins as could be expected if temperature was a cause of stress for copepods (Voznesensky et al. 2004; Petkeviciute et al. 2015). In a recent study, it was observed that under thermal stress, one species of copepod C. finmarchicus showed a strong response to temperature and duration of stress, involving up-regulation of genes related to protein folding, transcription, translation and metabolism, whereas in contrast, other species $C$. glacialis displayed only lowmagnitude changes in gene expression in response to temperature and duration of stress (Smolina et al. 2015). Therefore, absence of differential expression of genes related to thermal stress could be attributed to thermal acclimation and resilience of the Red Sea zooplankton population.

On the other hand UV indices in the region are very high, with values exceeding 11 from April to August (Fig. 1). Laboratory experiments have already shown that UV-B levels in the region in June increase the mortality of zooplankton in the Red sea (Al-Aidaroos et al. 2014, 2015). An increase in the expression of genes involved in oxidative stress for copepods indicates that it is one of the expected effects of UV-B (Lesser 2006). UV-B may also affect the immune system potentially leading to increase in the frequency of viral infections.

In July, an effect due to compounds released by Trichodesmium or increased UV-B or a combination of both fit the observations of a decline in surface abundance of copepods and appendicularia, and differential abundance of expressed genes related to oxidative stress and viral reproduction. A higher time resolution sampling during the spring and specific experiments will be needed to unravel whether there is a single main cause of mortality in play or mortality is due to a combination of factors. Predation is often thought to be the primary cause of copepod mortality (Hirst and Kiorboe 2002) but our observations of carcasses indicate that sources of mortality other than predation may have affected zooplankton population abundance. Indeed the metatranscriptomics data in this study have opened up the black box of non-predatory mortality in zooplankton and provided information to establish potential hypotheses for different factors at play.

\section{References}

Al-Aidaroos, A. M., M. M. O. El-Sherbiny, S. Satheesh, G. Mantha, S. Agustī, B. Carreja, and C. M. Duarte. 2014. High mortality of Red Sea zooplankton under ambient solar radiation. PLoS One 9: e108778. doi:10.1371/ journal.pone.0108778

Al-Aidaroos, A. M., M. M. O. El-Sherbiny, S. Satheesh, G. Mantha, S. Agustī, B. Carreja, and C. M. Duarte. 2015. Strong sensitivity of Red Sea zooplankton to UV-B radiation. Estuar. Coast. 38: 846-853. doi:10.1007/s12237-0149868-4
Denoeud, F., T. F. F. Ng, K. Rosario, J. G. Barbosa, A. M. Greco, M. Breitbart, and I. Hewson. 2010. Plasticity of animal genome architecture unmasked by rapid evolution of a pelagic tunicate. Science 330: 1381-1385. doi:10. 1126/science. 1194167

Dunlap, D. S., T. F. F. Ng, K. Rosario, J. G. Barbosa, A. M. Greco, M. Breitbart, and I. Hewson. 2013. Molecular and microscopic evidence of viruses in marine copepods. Proc. Natl. Acad. Sci. USA 110: 1375-1380. doi:10.1073/ pnas. 1216595110

Guo, C., and P. A. Tester. 1994. Toxic effect of the bloomforming Trichodesmium sp. (Cyanophyta) to the copepod Acartia tonsa. Nat. Toxins. 2: 222-227. doi:10.1002/nt.2620020411

Haas, B. J., and others. 2013. De novo transcript sequence reconstruction from RNA-Seq using the Trinity platform for reference generation and analysis. Nat. Protoc. 8: 1494-1512. doi:10.1038/nprot.2013.084

Hardy, J. T. 1982. The sea surface microlayer: Biology, chemistry and anthropogenic enrichment. Prog. Oceanogr. 11: 307-328. doi:10.1016/0079-6611(82)90001-5

Hawser, S. P., J. M. Oneil, M. R. Roman, and G. A. Codd. 1992. Toxicity of blooms of the cyanobacterium-Trichodesmium to zooplankton. J. Appl. Phycol. 4: 79-86. doi: 10.1007/BF00003963

Hewson, I., R. S. Poretsky, S. T. Dyhrman, B. Zielinski, A. E. White, H. J. Tripp, J. P. Montoya, and J. P. Zehr. 2009. Microbial community gene expression within colonies of the diazotroph, Trichodesmium, from the Southwest Pacific Ocean. ISME J. 3: 1286-1300. doi:10.1038/ismej.2009.75

Hirst, A. G., and T. Kiorboe. 2002. Mortality of marine planktonic copepods: Global rates and patterns. Mar. Ecol. Prog. Ser. 230: 195-209. doi:10.3354/meps230195

Ishii, S., S. Suzuki, T. M. Norden-Krichmar, A. Tenney, P. S. Chain, M. B. Scholz, K. H. Nealson, and O. Bretschger. 2013. A novel metatranscriptomic approach to identify gene expression dynamics during extracellular electron transfer. Nat. Commun. 4: 1601. doi:10.1038/ncomms2615

Karnovsky, M. J. 1961. Simple methods for "staining with lead" at high pH in electron microscopy. J. Biophys. Biochem. Cytol. 11: 729-732. doi:10.1083/jcb.11.3.729

Lesser, M. P. 2006. Oxidative stress in marine environments: Biochemistry and physiological ecology. Annu. Rev. Phys. 68: 253-278. doi:10.1146/annurev.physiol.68.040104.11 0001

Marchetti, A., and others. 2012. Comparative metatranscriptomics identifies molecular bases for the physiological responses of phytoplankton to varying iron availability. Proc. Natl. Acad. Sci. USA 109: E317-E325. doi:10.1073/ pnas. 1118408109

Mojib, N., M. Amad, M. Thimma, N. Aldanondo, M. Kumaran, and X. Irigoien. 2014. Carotenoid metabolic profiling and transcriptome-genome mining reveal functional equivalence among blue-pigmented copepods and 
appendicularia. Mol. Ecol. 23: 2740-2756. doi:10.1111/ mec. 12781

Ngugi, D. K., A. Antunes, A. Brune, and U. Stingl. 2012. Biogeography of pelagic bacterioplankton across an antagonistic temperature-salinity gradient in the Red Sea. Mol. Ecol. 21: 388-405. doi:10.1111/j.1365-294X. 2011.05378.x

O'Neil, J. M., and M. R. Roman. 1994. Ingestion of the cyanobacterium Trichodesmium spp. by pelagic harpacticoid copepods Macrosetella, Miracia and Oculosetella. Hydrobiologia 292: 235-240.

Petkeviciute, E., P. W. Kania, and A. Skovgaard. 2015. Genetic responses of the marine copepod Acartia tonsa (Dana) to heat shock and epibiont infestation. Aquacult. Rep. 2: 10-16. doi:10.1016/j.aqrep.2015.04.001

Radax, R., T. Rattei, A. Lanzen, C. Bayer, H. T. Rapp, T. Urich, and C. Schleper. 2012. Metatranscriptomics of the marine sponge Geodia barretti: Tackling phylogeny and function of its microbial community. Environ. Microbiol. 14: 1308-1324. doi:10.1111/j.1462-2920.2012.02714.x

Raitsos, D. E., I. Hoteit, P. K. Prihartato, T. Chronis, G. Triantafyllou, and Y. Abualnaja. 2011. Abrupt warming of the Red Sea. Geophys. Res. Lett. 38: L14601. Doi:10.1029/ $2011 \mathrm{gl} 1047984$

Roman, M. R. 1978. Ingestion of blue-green-alga Trichodesmium by harpactacoid copepod, Macrosetella gracilis. Limnol Oceanogr. 23: 1245-1248. doi:10.4319/lo.1978.23.6.1245

Smolina, I., S. Kollias, E. F. Møller, P. Lindeque, A. Y. M. Sundaram, J. M. O. Fernandes, and G. Hoarau. 2015. Contrasting transcriptome response to thermal stress in two key zooplankton species, Calanus finmarchicus and C. glacialis. Mar. Ecol. Prog. Ser.. 534: 79-93. doi:10.3354/ meps11398

Stewart, F. J., A. K. Sharma, J. A. Bryant, J. M. Eppley, and E. F. Delong. 2011. Community transcriptomics reveals universal patterns of protein sequence conservation in natural microbial communities. Genome Biol. 12: R26. doi:10.1186/gb-2011-12-3-r26

Urich, T., A. Lanzen, J. Qi, D. H. Huson, C. Schleper, and S. C. Schuster. 2008. Simultaneous assessment of soil microbial community structure and function through analysis of the meta-transcriptome. PLoS One 3: e2527. doi:10.1371/journal.pone.0002527

Voznesensky, M., P. H. Lenz, C. Spanings-Pierrot, and D. W. Towle. 2004. Genomic approaches to detecting thermal stress in Calanus finmarchicus (Copepoda: Calanoida). J. Exp. Mar. Biol. Ecol. 311: 37-46. doi:10.1016/j.jembe.2004.04. 017

Yadav, R. K., C. Bragalini, L. Fraissinet-Tachet, R. Marmeisse, and P. Luis. 2016. Metatranscriptomics of soil eukaryotic communities. Methods Mol. Biol. 1399: 273-287. doi:10. 1007/978-1-4939-3369-3_16

Zhang, G., and others. 2012. The oyster genome reveals stress adaptation and complexity of shell formation. Nature 490: 49-54. doi:10.1038/nature11413

\section{Acknowledgments}

We thank the captain and crew of the KAUST research vessel operated by the Coastal and Marine Resources Core lab (CMOR) at King Abdullah University of Science and Technology (KAUST) for their technical support during sampling. We are also thankful to the Analytical Core Lab and the Bioscience Core Lab at KAUST for their support and services. We also thank the two anonymous reviewers for their helpful comments. This research was supported by baseline funding provided by KAUST to Prof. Xabier Irigoien.

\section{Conflict of Interest}

None declared.
Submitted 16 February 2016 Revised 24 June 2016 Accepted 25 July 2016 